\title{
Desarrollo larval de Mursia gaudichaudi (Milne Edwards 1837) (Decapoda, Brachyura, Calappidae)
}

\section{Larval development of Mursia gaudichaudi (Milne Edwards 1837) (Decapoda, Brachyura, Calappidae)}

\author{
María de los A. Gallardo ${ }^{1 *}$ \& Armando Mujica ${ }^{1 *}$ \\ ${ }^{1}$ Facultad de Ciencias del Mar, Universidad Católica del Norte. Casilla 117, Coquimbo Chile. \\ *Email: amujica@ucn.cl, maryangelgs@gmail.com
}

\begin{abstract}
RESUMEN
Se describe el desarrollo larval de Mursia gaudichaudi basado en las zoeas I y II cultivadas en laboratorio y las zoeas I a la IV obtenidas de muestras planctónicas. Las larvas cultivadas en laboratorio, se obtuvieron de hembras ovígeras capturadas en la costa de Coquimbo, norte de Chile. Las características morfológicas y morfométricas de éstas permitieron identificar las larvas capturadas con redes de plancton en la zona de Caldera y Coquimbo, durante la primavera de 2005. Las espínulas en la región proximal de la espina rostral, las de las espinas laterales del cefalotórax, los procesos espinosos de la antena y la longitud e inserción de las espinas laterales del abdomen, son las principales características morfológicas y morfométricas que permiten diferenciarlas de las restantes especies de la superfamilia Calappoidea (Calappidae y Hepatidae).
\end{abstract}

Palabras clave: Brachyura, Calappidae, Coquimbo, Zoea.

\begin{abstract}
The larval development of Mursia gaudichaudi is described based on the laboratory cultivated zoeae in stages I and II, and plankton samples of zoeae from stages I to IV. The laboratory-reared larvae were obtained from ovigerous females captured off the coast of Coquimbo, northern Chile. Their morphological and morphometric characteristics allowed identifying the larvae captured with plankton nets in the area of Caldera, and Coquimbo, northern Chile, during spring time of year 2005. The espinule in the proximal region of the rostral spine, those of lateral carapace spines, the spiny processes of the antenna, and the length and insertion of the lateral abdominal spines are the principal characteristics that allow differentiating them from the remaining species of the superfamily Calappoidea (Calappidae and Hepatidae).
\end{abstract}

KEYwords: Brachyura, Calappidae, Coquimbo, Zoea.

\section{INTRODUCCIÓN}

Mursia gaudichaudi (Milne Edwards 1837) es uno de los componentes más frecuentes y abundantes en la fauna acompañante de la pesquería de arrastre de camarones y langostinos que se efectúa entre la II y VIII Región de Chile (Acuña et al. 2002). En este tipo de pesquería esta especie carece de importancia económica directa (Henríquez \& Bahamonde 1976), pero según Hendrickx (1995), en el norte de Chile y el sur de Perú se le explota localmente y es comercializada en fresco. Tresierra \& Arana (1988) señalan que esta especie es alimento de importantes recursos pesqueros de valor comercial.
M. gaudichaudi pertenece a la familia Calappidae (Garth 1957), la cual está compuesta por especies principalmente tropicales y algunos representantes de ambientes templados (Jesse \& Stotz 2003). Se distribuye desde el golfo de Farellones, California, hasta Talcahuano, Chile (Retamal 1981, 2000) y es la única especie de esta familia que habita en el mar de Chile. Antezana et al. (1965) han reportado su presencia en Chiloé y Garth (1957) en las islas Galápagos, por lo que se considera una especie de amplia distribución. Su distribución batimétrica ha sido descrita por diversos autores en rangos comprendidos entre 0 y $550 \mathrm{~m}$ de profundidad (Garth 1957, Antezana et al. 1965, Arana et al. 1975, Retamal 2000). Según Menares (2002), es más 
abundante a mayores profundidades, donde predomina el sustrato fino, altas salinidades y bajas concentraciones de oxígeno, lo que contrasta parcialmente con lo descrito por Jesse \& Stotz (2003), quienes la encontraron preferentemente sobre fondos fangosos en áreas someras. Según Tresierra \& Arana (1988), la proporción de hembras predomina sobre los machos durante todo el año $(6,5: 1$ entre 0 a $400 \mathrm{~m}$ de profundidad), con mayor cantidad de hembras ovígeras en octubre, declinando en noviembre. Estos autores, señalan que la liberación de larvas ocurre entre octubre y noviembre. Retamal (2000), indica que las hembras ovígeras abundan entre 4 y $5 \mathrm{~m}$ de profundidad. El desarrollo larval de los Brachyura está constituido por prezoea, zoea y megalopa, cuyo número de estadios, depende de la especie y las condiciones ambientales a las que estén sometidas (Boschi et al. 1969, Barría et al. 2005).

De la familia Calappidae, sólo han sido descritas las primeras zoeas de seis especies pertenecientes al género Calappa, cuya morfología es comparada por Taishaku \& Konishi (1995). Además, se ha descrito el desarrollo larval de tres especies de la familia Hepatidae, también de la superfamilia Calappoidea de las cuales Pohle et al. (1999), discuten comparativamente la morfología de las primeras zoeas de Hepatus pudibundus y Calappa gallus. En Chile, Fagetti (1960) describe la primera zoea de Hepatus chilensis, cuya morfología larval posteriormente fue comparada con la de Hepatus epheliticus por Taishaku \& Konishi (1995). Respecto del desarrollo larval de las especies del género Mursia, no existen antecedentes a nivel mundial.

La información meroplanctónica generada paralelamente del estudio del recurso camarón y langostinos (Heterocarpus reedi, Cervimunida johni y Pleuroncodes monodon) (Mujica y Acuña en ed.) ha permitido conocer aspectos relacionados con la distribución y abundancia de las larvas de Mursia gaudichaudi (Gallardo 2007). Al respecto, Palma (1976) y Bryars \& Havenhand (2004), han señalado que este tipo de información es relevante en el conocimiento de las áreas y época de desove de los progenitores.

De acuerdo a los antecedentes anteriormente expuestos, el desarrollo larval de $M$. gaudichaudi que se describe constituye un aporte al conocimiento del decápodo más abundante de la fauna acompañante de las pesquerías bentónicas de la zona y un potencial recurso pesquero, de cuyo género se desconocen sus larvas.

\section{MATERIALES Y MÉTODOS}

Hembras ovígeras de $M$. gaudichaudi, capturadas mediante arrastres de fondo ( $80 \mathrm{~m}$ de profundidad) frente a Punta Teatinos $\left(29^{\circ} 47,9^{\prime} \mathrm{S}-71^{\circ} 19,75^{\prime} \mathrm{W}\right)$, se mantuvieron en acuarios de agua de mar, microfiltrada $(1 \mu \mathrm{m})$, tratada con luz UV y aeración constante, hasta el momento de la eclosión de las larvas.

Diez larvas recién eclosionadas se mantuvieron en recipientes de $250 \mathrm{~mL}$ de capacidad con $200 \mathrm{~mL}$ de agua de mar microfiltrada, tratada con luz $\mathrm{UV}$, con aeración constante y a temperatura de $14^{\circ} \mathrm{C} \pm 0,5^{\circ} \mathrm{C}$. Diariamente se determinó la mortalidad, la presencia de mudas y el estado de desarrollo de las larvas. Con igual frecuencia se cambió el agua de los recipientes, previo a la alimentación, consistente en $0,5 \mathrm{~mL}$ de Isochrysis galbana y Nanocloris spp. (1:1).

Las descripciones de los primeros estados de desarrollo (zoeas I y II) obtenidas en laboratorio permitió la identificación de las larvas de las muestras planctónicas provenientes de los cruceros FIP N ${ }^{\circ}$ 2004-45, efectuados en octubre y diciembre del 2005, en el sistema de bahías de Caldera (26 46' $\left.27^{\circ} 08^{\prime} \mathrm{S}\right)$ y en la zona de Coquimbo $\left(29^{\circ} 21^{\prime}-30^{\circ} 15^{\prime} \mathrm{S}\right)$. Los caracteres morfológicos de las zoeas cultivadas I y II se buscaron y reconocieron en las larvas de igual desarrollo capturadas en el plancton, los cuales no tuvieron variaciones en forma, inserción, setación, número de espinas ni tamaño. La persistencia de los caracteres diagnósticos en los siguientes estados de desarrollo permitió la identificación de las zoeas III y IV provenientes de las muestras planctónicas. Entre estos caracteres destacan las espínulas en las espinas laterales y rostral del cefalotórax. Esto, sumado a la morfología típica del telson de las larvas de la familia Calappidae (Seridji 1993; Guerao et al. 1999) y dado que $M$. gaudichaudi es la única especie de esta familia, permitió certeza en la identificación de las larvas planctónicas.

La observación de los diferentes estados de desarrollo se efectuó mediante el uso de un microscopio estereoscópico provisto de un tubo de dibujo, que permitió la diagramación y medición de las larvas. Para el análisis comparativo de las larvas respecto de las especies de la familia, se consideró la longitud total del caparazón (LT), como la distancia comprendida entre el ápice de la espina rostral y dorsal.

\section{RESULTADOS}

Entre los caracteres más importantes para la identificación de las larvas de $M$. gaudichaudi, y su secuencia de desarrollo, se consideró la presencia de pequeñas espinas (espínulas) en el margen dorsal de las espinas laterales y en la región distal de la espina rostral, cuyo número y tamaño dependen del estado de desarrollo.

ZoEA I

La longitud total promedio es de 1,7 $\pm 0,07 \mathrm{~mm}(\mathrm{~N}=10)$. El caparazón tiene una espina dorsal curvada dorsalmente, 
de longitud levemente mayor que el largo del cefalotórax. A ambos lados de su base se ubica una pequeña seta. La espina rostral es de similar longitud que la espina dorsal, con 4 espínulas a cada lado de la región distal y 3 en la parte anteroproximal, de menor tamaño que las laterales. Las espinas laterales son de inserción media, de longitud similar al ancho del cefalotórax, levemente curvadas anteroventralmente y con 4 a 5 espínulas prominentes en su margen dorsal (Fig. 1a).

Las anténulas son unirramias, con exopodito de un solo artículo y 4 astetascos apicales de similar longitud (Fig. 2a). Las antenas tienen forma de espina, de similar longitud que la espina rostral y con 10 a 11 espínulas prominentes en el contorno distal. El endopodito es corto como una pequeña espínula (Fig. 2e).
El coxopodito de la maxílula tiene 6 setas plumosas de longitud similar entre ellas. Basipodito con 4 setas apicales plumosas más anchas y largas que las del coxopodito y una subapical de menor tamaño. Endopodito con dos artículos, una seta proximal en el primer artículo y con 4 setas plumosas apicales en el artículo distal (Fig. 3a).

Maxila con coxopodito, basipodito y endopodito bilobulados y con setas plumosas. El coxopodito con $3+2$ setas apicales. Basipodito con $3+3$ setas apicales y $1+1$ subapicales. Endopodito con $2+3$ setas apicales y 1 subapical. Escafognatito con 4 setas más largas que las restantes de la maxila y una larga proyección apical carente de setas. Las 3 primeras setas distales son plumosas (Fig. 4a).
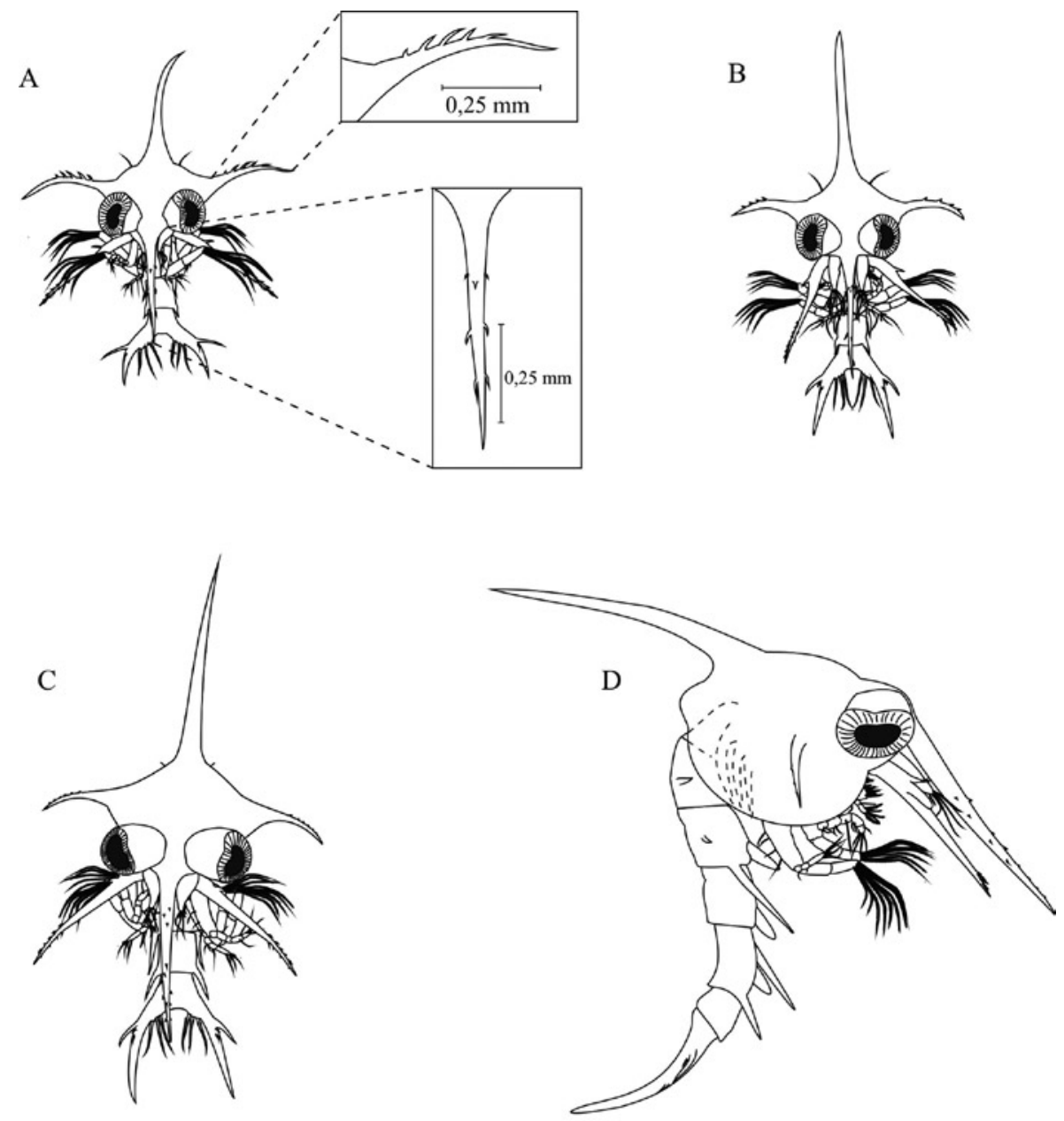

$1,0 \mathrm{~mm}$

FIGURA 1. Zoeas de M. gaudichaudi. a, b y c, vista frontal de zoeas I; II y III. d, vista lateral de zoea IV.

FIGURE 1. Zoeae of M. gaudichaudi. a, b and c, front view of zoeae I; II and III. d, lateral view of zoea IV. 
Gayana 74(1), 2010

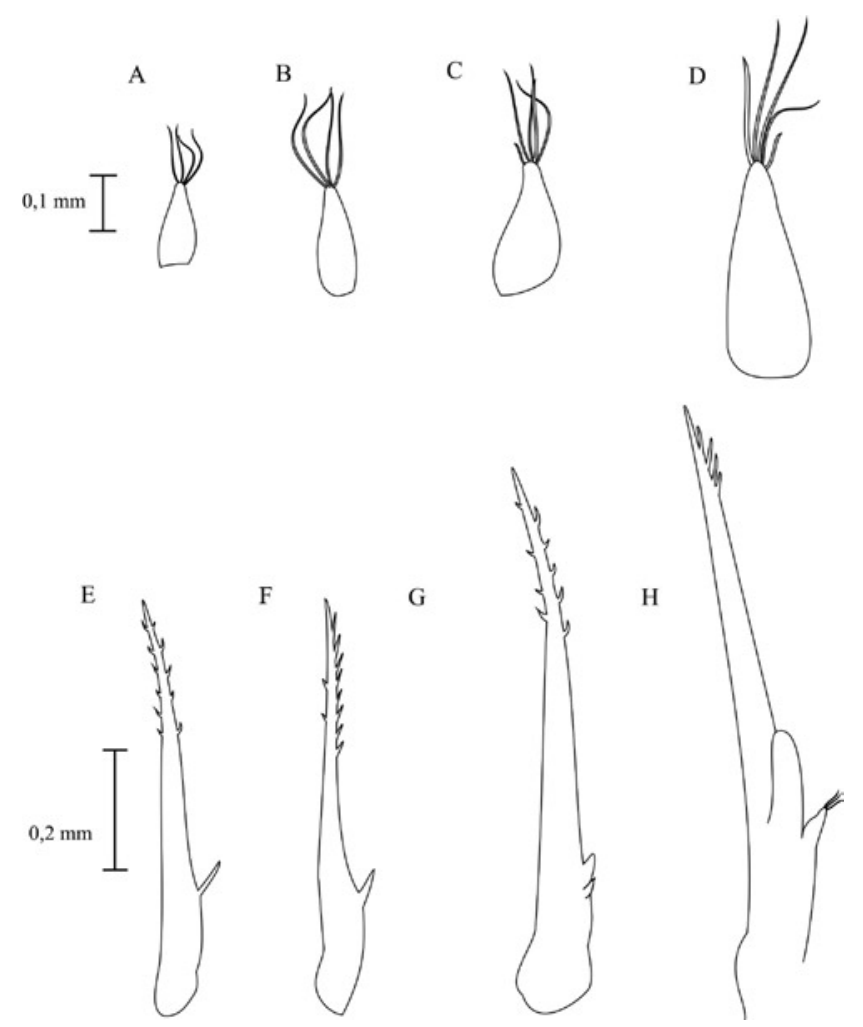

Figura 2. Anténula y antena de M. gaudichaudi. a - d: anténula de zoea I - IV. e - h: antena de zoea I - IV.

FIGURE 2. Antennule and antenna of M. gaudichaudi. a - d: antennule of zoea I - IV. e - h: antenna of zoea I - IV.

A
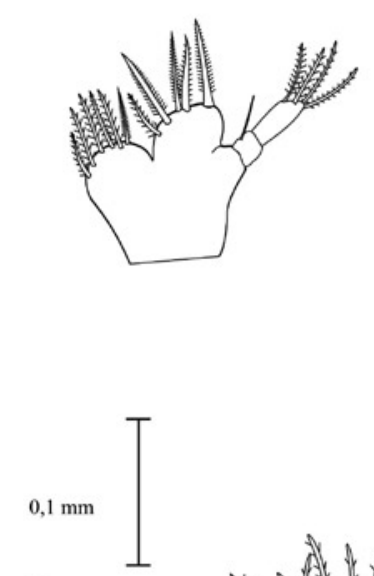

C

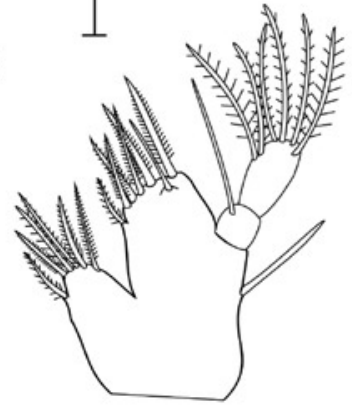

B
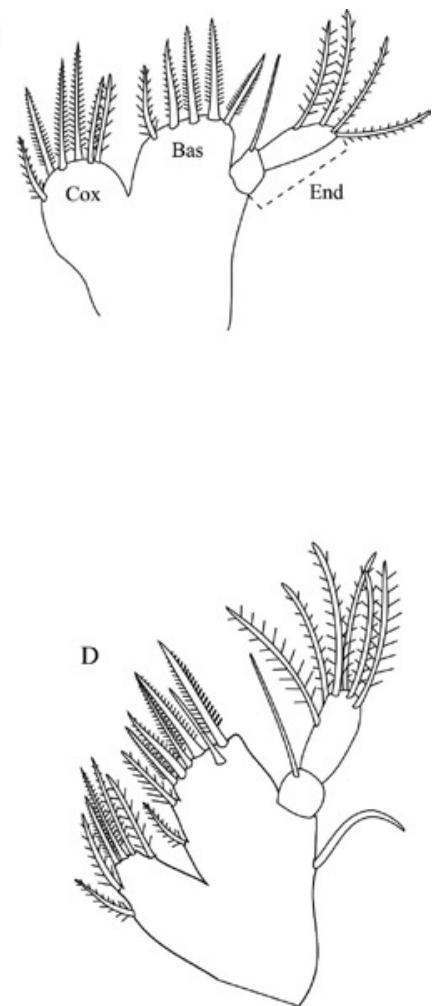

FIgura 3. Maxílulas de M. gaudichaudi. a - d: zoea I - IV. Cox: Coxopodito; Bas: Basipodito; End: Endopodito.

FIgURE 3. Maxillule of M. gaudichaudi. a - d: zoea I - IV. Cox: Coxalendite; Bas: Basalendite; End: Endopod. 
A
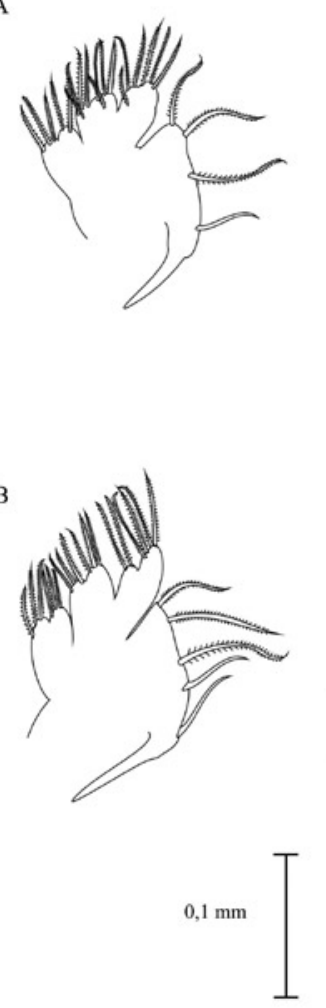

C
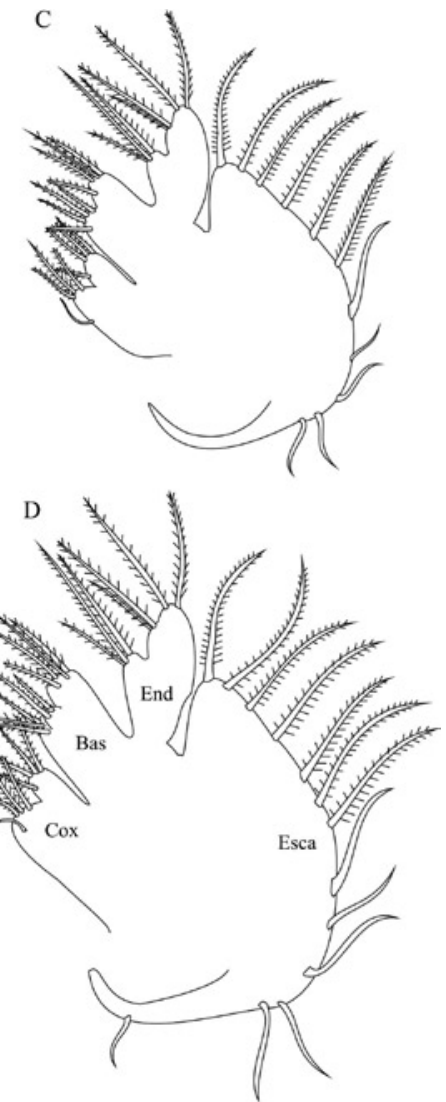

FIgURA 4. Maxila de M. gaudichaudi. a - d: zoea I - IV. Cox: Coxopodito; Bas: Basipodito; End: Endopodito; Esca: Escafognatito.

FIgURE 4. Maxilla of M. gaudichaudi. a - d: zoea I - IV. Cox: Coxalendite; Bas: Basalendite; End: Endopod; Esca: Scaphognathite.

El primer maxilípedo tiene 3 setas en el margen posterior del basipodito. Endopodito está compuesto por 5 artículos de similares longitudes y setación $1+2+1+2+4$. Exopodito con dos artículos de longitud similar a la mitad del basipodito. El artículo proximal sin setas y el distal con 4 setas plumosas apicales de similares longitudes (Fig. 5a).

El basipodito del segundo maxilípedo tiene 4 setas en el margen posterior. El endopodito tiene 3 artículos, que en conjunto no superan la mitad de la longitud del basipodito. El primer y segundo artículo tienen una seta y el tercero con 4 apicales más una seta subapical, de longitud superior a las apicales. El exopodito tiene dos artículos de longitud similar a la mitad del basipodito. El proximal sin setas y el distal con 4 setas plumosas apicales de similares longitudes (Fig. 5e).

El abdomen esta conformado por 5 somitos. El segundo y tercer somito tienen espinas laterales de inserción media. Las espinas del segundo somito, curvadas hacia delante y las del tercero hacia atrás. Los somitos tercero al quinto tienen procesos laterales distales de longitud similar a la mitad del siguiente somito (Fig. 6a).

El telson tiene una rama furcal a cada lado y una espina lateral externa en cada una de ellas, que es prominente. En las ramas furcales tienen una pequeña espina dorsal y entre ellas 6 setas plumosas $(3+3)$ (Fig. 6a). 
Gayana 74(1), 2010

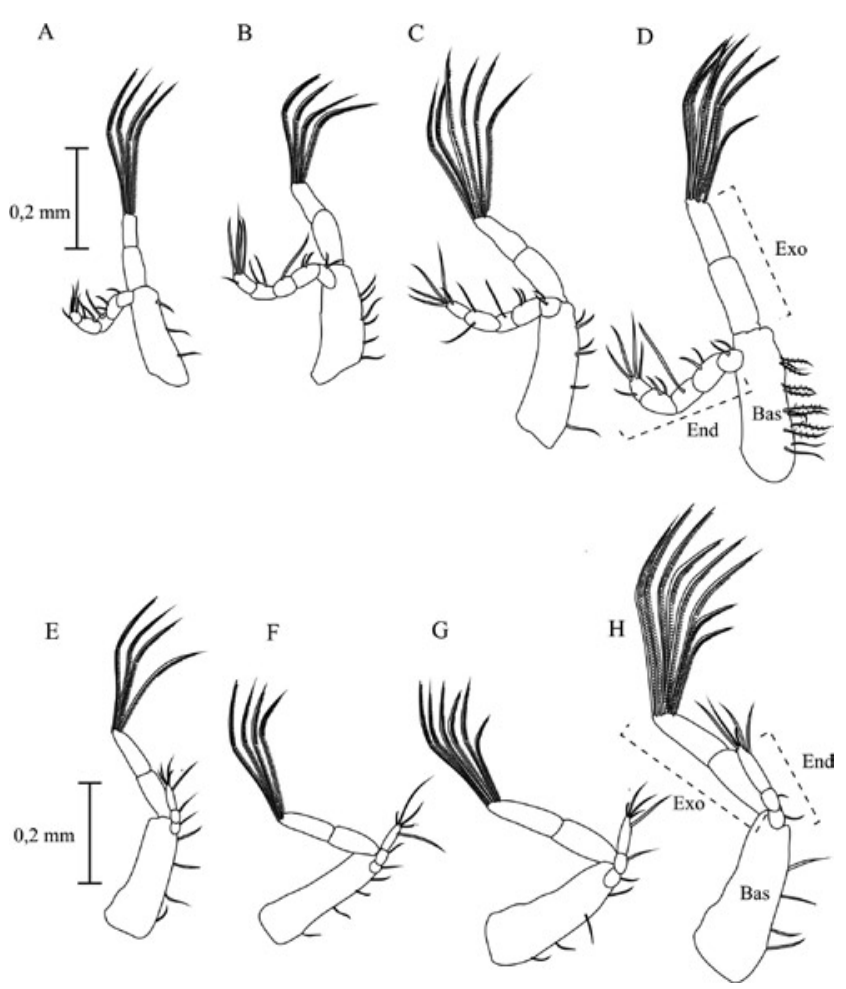

FIgURA 5. Primer y segundo maxilípedo de M. gaudichaudi. a - d: primer maxilípedo de zoea I - IV. e - h: segundo maxilípedo de zoea I - IV. Bas: Basipodito; End: Endopodito; Exo: Exopodito.

FIGURE 5. First and second maxilliped of M. gaudichaudi. a - d: first zoea maxilliped I - IV. e - h: second zoea maxilípedo I - IV. Bas: Basalendite; End: Endopod; Exo: Exopod.
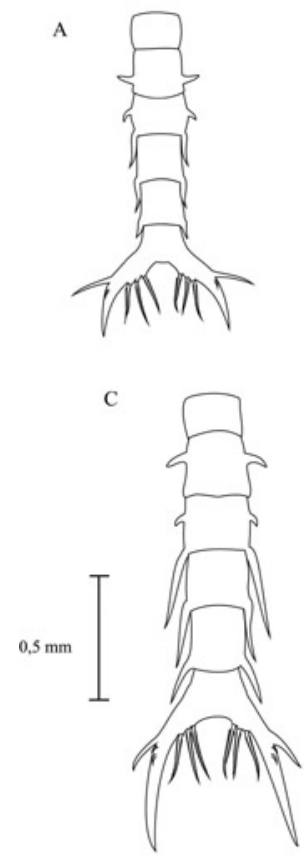

Figura 6. Abdomen de M. gaudichaudi. a - d: zoea I - IV.
B
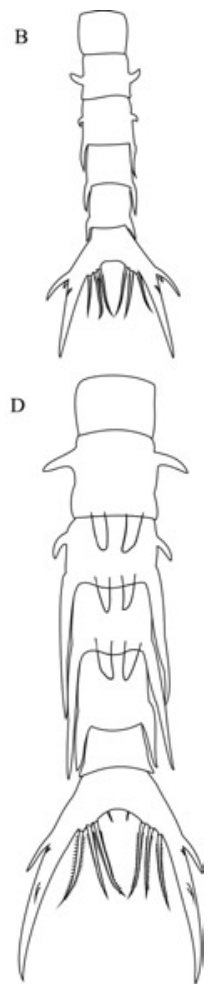

FIgURE 6. Abdomen of M. gaudichaud. a - d: zoea I - IV. 


\section{ZoEA II}

La longitud total promedio es de 2,0 $\pm 0,02 \mathrm{~mm}(\mathrm{~N}=10)$. Se obtuvieron las primeras zoea II a 10 días de la eclosión. La morfología general del cefalotórax es similar al de las zoeas I, aunque de mayor tamaño. Las espínulas ubicadas sobre las espinas laterales del cefalotórax son de menor tamaño que en la zoea I y de inserción más distal (Fig. 1b). Las anténulas mantienen el mismo número de astetascos apicales que el estadio anterior (Fig. 2b) y las antenas (Fig. 2f) aumentan su denticulación sólo en uno de sus márgenes laterales. El endopodito de la antena es más grueso que en la zoea I.

En este estadio, las maxílulas mantienen la setación descrita para zoea I, pero la seta ubicada en el primer artículo del endopodito tiene mayor longitud, siendo casi tan larga como las setas del basipodito (Fig. 3b).

El coxopodito de la maxila tiene $3+3$ setas plumosas apicales. El basipodito mantiene setación del estadio anterior. El endopodito tiene $2+4$ setas apicales y $1+$ 0 subapicales. El escafognatito con 5 setas, de las cuales las 3 distales son plumosas y un tercio más largas que las restantes setas de la maxila (Fig. 4b).

El basipodito del primer maxilípedo tiene $1+1+2+2$ setas de similar longitud entre ellas, en el margen posterior. El endopodito esta compuesto por 5 artículos, cuya setación es $2+2+1+2+5$. La seta del tercer artículo es tan larga como las setas apicales del quinto. El exopodito conserva la setación de zoea I (Fig. 5b).

Segundo maxilípedo conserva morfología encontrada en zoea I, pero las setas apicales del tercer artículo son casi dos veces más largas que las del basipodito (Fig. 5f).

El abdomen no posee variación con respecto al estadio anterior. El telson tiene una pequeña espina entre las espinas laterales y las ramas furcales. Las ramas furcales son más largas y rectas que el estadio anterior. El resto del telson se mantiene sin variación (Fig. 6b).

\section{ZoEA III}

La longitud total promedio es de 2,8 $\pm 0,24 \mathrm{~mm}(\mathrm{~N}=10)$. Las setas de la base de la espina dorsal son menos notorias que en estadios anteriores. Las espinas laterales poseen 5 a 6 espínulas sobre el margen dorsal, de inserción distal y más pequeñas que en zoea II. La espina rostral tiene 9 a 10 pequeñas espinas en su región distal y 3 pequeñas en la región proximal, de mayor tamaño que las encontradas en espinas laterales (Fig. 1c).

Las anténulas tienen 5 astetascos apicales. El proximal, de longitud inferior a la mitad de los restantes (Fig. 2c). Endopodito de la antena, digitiforme. El exopodito es incipiente con 2 a 3 pequeñas setas apicales, de similar longitud (Fig. 2g).

El coxopodito de la maxílula tiene 6 setas plumosas. El basipodito posee 5 setas plumosas apicales y dos subapicales. Endopodito con una seta de inserción media en primer artículo de tamaño similar a la longitud total del endopodito. El segundo artículo del endopodito tiene 6 setas plumosas apicales, de longitud similar a la del endopodito. Exopodito con una seta marginal de similar longitud que las del endopodito (Fig. 3c).

El coxopodito de la maxila tiene $3+3$ setas plumosas apicales y $1+1$ subapical. El basipodito y endopodito conservan la setación del estadio anterior. El escafognatito tiene 11 setas. Las 6 distales son plumosas y más largas que las del endopodito (Fig. 4c).

El primer maxilípedo, tiene 6 setas plumosas en el segundo artículo del exopodito. El resto de sus estructuras carecen de modificaciones importantes respecto del estadio anterior (Fig. 5c). El segundo maxilípedo tiene 6 setas plumosas apicales en el segundo artículo del exopodito. El resto de sus estructuras conservan la setación de zoea II (Fig. 5g).

Desde el tercer al quinto somito del abdomen tienen procesos laterales distales. La longitud del proceso lateral del tercer somito alcanza la mitad del quinto somito. Los procesos de los somitos cuarto y quinto son tan largos como el cuarto somito. Las ramas furcales del telson son de longitud dos veces mayor a las setas interiores del telson. El resto de las estructuras del telson y abdomen mantienen la morfología del estadio anterior (Fig. 6c). Los pereiópodos son digitiformes e incipientes en este estadio.

\section{Zoea IV}

La longitud total promedio es de 3,6 $\pm 0,13 \mathrm{~mm}(\mathrm{~N}=5)$. La espina dorsal del cefalotórax está pronunciadamente curvada hacia atrás. Las espinas laterales tienen una longitud similar a la mitad de la espina rostral, las cuales poseen 3 a 4 espínulas de inserción distal sobre el margen dorsal y más pequeñas que en zoea III. La espina rostral conserva el número de espínulas, pero de menor tamaño que zoea III (Fig. 1d).

La anténula conserva la setación del estadio anterior (Fig. 2d). Los procesos espinosos de la antena están sólo armados en un margen lateral con 4 a 5 espinas. El endopodito duplica en tamaño al exopodito. Este último tiene 3 a 4 setas apicales (Fig. 2h).

La setación del coxopodito, endopodito y exopodito de la maxílula es similar al estadio anterior. El basipodito posee 6 setas plumosas apicales y dos subapicales (Fig. 3d). 
El coxopodito y endopodito de la maxila conservan la setación del estadio anterior. El basipodito con $4+3$ setas plumosas apicales y $1+1$ subapical. El escafognatito tiene 12 setas. Las 7 distales plumosas y más largas que las 5 proximales que no son plumosas. La proyección apical del escafognatito tiene una seta marginal (Fig. 4d).

Primer maxilípedo sin modificaciones importantes respecto del estadio anterior. Basipodito del primer maxilípedo con $2+2+3+2+3$ setas plumosas en el margen posterior. El endopodito tiene una seta en su tercer artículo, de longitud dos veces mayor a las del artículo distal. El exopodito tiene 7 setas plumosas apicales en el artículo distal (Fig. 5d).

El segundo artículo del exopodito del segundo maxilípedo tiene 8 setas plumosas apicales. Las dos proximales de menor longitud que las restantes. El resto de sus estructuras conservan la setación de estadio anterior (Fig. 5h).

El abdomen esta constituido por 6 somitos. Los procesos laterales de los somitos 3, 4 y 5 son de longitud similar al doble del largo de un somito abdominal. El sexto segmento abdominal carece de procesos laterales (Fig. 6d). Los pleópodos son incipientes en los somitos 2, 3 y 4 (Fig. $1 \mathrm{~d}$ y $6 d)$. El telson tiene setación $4+4$. Las dos setas proximales son incipientes (Fig. 6d).

\section{DISCUSIÓN}

Las larvas de $M$. gaudichaudi tienen la morfología típica de las larvas de Brachyura, caracterizada por un cefalotórax globoso, provisto de una espina dorsal, una rostral y dos laterales (Fagetti 1960; Martin 1984; Pohle et al. 1999). El telson es similar a los descritos para la familia Calappidae (Fagetti 1960; Pohle et al. 1999).

El cefalotórax de la zoea I de $M$. gaudichaudi es muy similar al de Calappa granulata (Guerao et al. 1999), aunque existen diferencias en la distribución y cantidad de espínulas de las espinas laterales y rostral. M. gaudichaudi posee 3 espínulas pequeñas en la región proximal de la espina rostral y prominentes espínulas en el margen dorsal de las espinas laterales, mientras que en las larvas de C. granulata todas las espínulas de la espina rostral son de igual tamaño y las del margen dorsal de las espinas laterales son de menor tamaño, similares a las de las zoeas de $H$. chilensis. La setación de los apéndices bucales de las zoeas de la superfamilia Calappoidea es característica de cada género (Taishaku \& Konishi 1995), por lo que la setación encontrada en las zoeas I de $M$. gaudichaudi fue un criterio determinante en su identificación (Tabla 1).

La zoea I de $M$. gaudichaudi posee telson y abdomen similares a los descritos para zoeas de la superfamilia Caloppoidea (Fagetti 1960, Seridji 1993, Taishaku \& Konishi 1995, Guerao et al. 1999, Pohle et al. 1999), sin embargo las espinas laterodistales de los somitos abdominales de las larvas de $M$. gaudichaudi son mucho más largas que las de las zoeas I del resto de la superfamilia. Además, la segunda espina lateral del telson está ausente en zoea I, a diferencia de lo que ocurre en el resto de las larvas de la superfamilia.

Los caracteres que diferencian a la zoea I de esta especie, del resto de las zoeas de la superfamilia Calappoidea, son la presencia de prominentes espínulas en el margen dorsal de las espinas laterales del cefalotórax, la presencia de 3 pequeñas espínulas en la región proximal de la espina rostral, los procesos espinosos de la antena, que son de similar longitud que la espina rostral y los procesos laterales del abdomen, que alcanzan la mitad de la longitud que el siguiente somito son determinantes para la identificación de las zoeas I. Estas características se mantienen en los siguientes estados de desarrollo, obtenidos tanto del plancton como en laboratorio, lo cual concuerda con lo señalado por Guerao et al. (2008), quienes encuentran igual morfología y tamaño de las larvas de Maja brachydactyla, cultivadas y obtenidas del plancton. Al respecto, Dornelas et al. (2003) obtuvieron la primera zoea de Hymenosoma orbiculare en laboratorio, completando su desarrollo con las larvas obtenidas del plancton, basados en caracteres morfológicos persistentes en ellas.

Los conspicuos caracteres morfológicos anteriormente descritos, observados en las primeras zoeas (I y II) cultivadas en laboratorio y que se mantienen en los ejemplares obtenidos en el plancton, tanto en iguales estadios como más avanzados, permitieron describir el desarrollo larval de la especie, que es el primero efectuado para género a nivel mundial. Además, éstos permiten distinguir a las zoeas de $M$. gaudichaudi de las larvas de Brachyura descritas para la zona de estudio. 
Desarrollo larval de Mursia gaudichaudi: María de los Ángeles Gallardo S. \& Armando Mujica R.

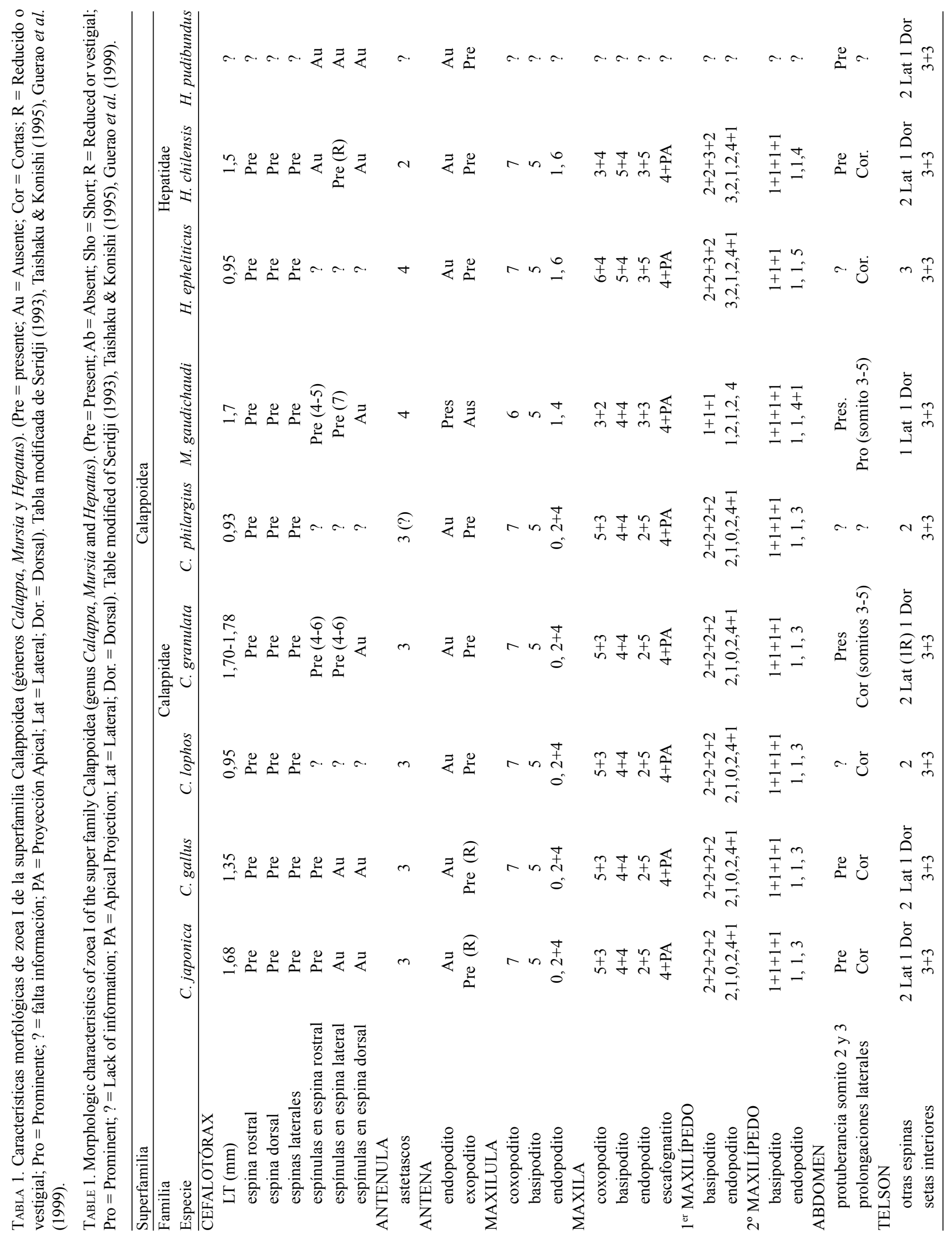




\section{AGRADECIMIENTOS}

Los autores agradecen a la tripulación de L/O Stella Maris de la Universidad Católica del Norte, que colaboraron en el muestreo y a los colegas Andrés González, Natalia Ottone y Ángelo Navarro, que contribuyeron en la separación de las muestras zooplanctónicas.

\section{BIBLIOGRAFÍA}

AcuÑa, E., L. Cid, S. ZúÑIgA, K. SoRia \& A. Cortés. 2002. Pesca experimental de crustáceos con trampas entre la II y IV Regiones. Informes Técnicos FIP. FIP/IT No 2001-22169.

Antezana, T., E. Fagetti \& M.T. López. 1965. Observaciones bioecológicas en decápodos comunes de Valparaíso. Revista de Biología Marina, Valparaíso 12:1-60.

Arana, P., T. Melo, L. Noziglia, I. Sepúlveda, N. Silva, G. Yany \& E. YÁÑEz. 1975. Los recursos demersales de la Región de Valparaíso, Chile. Revista de la Comisión Permanente del Pacífico Sur 3:39-61.

Barría, E.M., C.G. Jara \& K.A. PaschKe. 2005. La temperatura como factor de variabilidad en el desarrollo y morfología de larvas zoeas de Acanthocyclus hassleri Rathbun (Decapoda, Brachyura, Atelecyclidae) cultivadas en laboratorio. Investigaciones Marinas, Valparaíso 33(1):25-41.

Boschi, E.E., M.A. Scelzo \& B. Goldstein. 1969. Desarrollo larval del cangrejo, Halicarcinus planatus (Fabricius) (Crustacea, Decapoda, Hymenosomidae), en el laboratorio, con observaciones sobre la distribución de la especie. Bulletin of Marine Science 19(1):225-242.

Bryars, S. \& J. HaVenhand. 2004. Temporal and spatial distribution and abundance of blue swimmer crab (Portunus pelagicus) larvae in a temperature gulf. Marine and Freshwater Research 55(8):809-818.

Dornelas, M., J. Paula \& A. Macia. 2003. The larval development of Hymenosoma orbiculare Desmarest, 1825 (Crustacea: Decapoda: Brachyura: Hymenosomatidae). Journal of Natural History 37:2579-2597.

FagetTI, E. 1960. Primer estadio larval de cuatro crustáceos braquiuros de la bahía de Valparaíso. Revista de Biología Marina, Valparaíso. 10:143-154.

Gallardo, M.A. 2007. Descripción, distribución y abundancia de larvas de Mursia gaudichaudi (H. Milne Edwards, 1837) (Decapoda, Brachyura, Calappidae) en la III y IV Región $\left(26^{\circ} 45^{\prime}-30^{\circ} 10^{\prime} \mathrm{S}\right)$, Chile. Tesis para optar al título de Biólogo Marino. Universidad Católica del Norte. Coquimbo, Chile. 88 pp.

Garth, J.S. 1957. The Crustacea Decapoda Brachyura of Chile. Reports of the Lund University Chile Expedition 19481949, 29. Lunds Universitets Arsskrift. N. F. 57:1-130.

Guerao, G., P. Abello \& P. Torres 1999. Morphology of the first zoea of the Shamefaced Crab Calappa granulata (Brachyura, Calappidae) obtained in the laboratory. Butlleti de la Institucio Catalana d'Historia Natural 67:39-44.
Guerao, G., E. Pastor, J. Martin, M. Andrés, A. Estévez, A. Grau, J. DuRÁn \& G. Rotllant. 2008. The larval development of Maja squinado and M. brachydactyla (Decapoda, Brachyura, Majidae) described from plankton collected and laboratory-reared material. Journal of Natural History 42(33-34):2257-2276.

Hendrickx, M.E. 1995. Cangrejos. En: Guía FAO para la identificación de especies para los fines de pesca, Pacífico centro-oriental, plantas e invertebrados (Eds Fisher, W., Krupp, F., Schneider, W., Sommer, C., Carpenter K. \& Niem, V.H.), pp 646. Organización de las Naciones Unidas para la Agricultura y la Alimentación, Roma.

Henríquez, G. \& N. Bahamonde. 1976. Clave de identificación y datos biológicos de jaibas y pancoras frecuentes en las pescas comerciales de Chile (Crustacea, Decapoda, Brachyura). Instituto de Fomento Pesquero, Santiago (Chile) Investigaciones Pesqueras 21:1-73.

Jesse, S. \& W. Stotz. 2003. Spatio-temporal distribution patterns of the crab assemblage in the shallow subtidal of the north Chilean Pacific coast. Crustaceana 75:1161-1200.

Martin, J.W. 1984. Notes and bibliography on the larvae of xanthid crabs, with a key to the known xanthid zoeas of the Western Atlantic and Gulf of Mexico. Bulletin of Marine Science 34(2):220-239.

Menares, B. 2002. Estructura comunitaria de peces y crustáceos bentodemersales en relación a la hidrografia y sustrato en la zona centro sur de Chile. Tesis para optar al título de Oceanógrafo. Universidad Católica de Valparaíso. Valparaíso, Chile. 102 pp.

MujicA, A., E. AcuÑa \& M.L. Nava (en ed). Distribución y abundancia de larvas de Heterocarpus reedi Bahamonde, 1955, Cervimunida johni Porter, 1903 y Pleuroncodes monodon H. Milne Edwards, 1837, en la zona de Coquimbo y Caldera, Chile. Latin American Journal of Aquatic Research.

Palma, S. 1976. Meroplancton de la Región de Valparaíso. Ciencia y Tecnología del Mar 2:99-116.

Pohle, G., F.L. Mantelatto, M.L. Negreiros-fransozo \& A. Fransozo. 1999. Larval Decapoda (Brachyura). En: South Atlantic Zooplankton. (Ed. Boltovskoy D), pp 1281-1351. Leiden, The Netherlands.

Retamal, M.A. 1981. Catálogo ilustrado de los crustáceos decápodos de Chile. Gayana 44:1-110.

Retamal, M.A. 2000. Decápodos de Chile. 1995. World Biodiversity Database CD-ROM Series. ETI-U. Concepción. SpringerVerlag.

SERIDJI, R. 1993. Descriptions of some planktonic larvae of the Calappidae (Crustacea, Decapoda, Brachyura). Journal of Plankton Research 15(4):437-453.

Taishaku, H. \& K. Konishi. 1995. Zoeas of Calappa species with special reference to larval characters of the family Calappidae (Crustacea, Brachyura). Zoological Science 12(5):649-654.

Tresierra, A. \& P. Arana. 1988. Observaciones biológicas sobre Mursia gaudichaudi (H. Milne Edwards, 1837) desde Valparaíso, Chile (Decapoda, Brachyura, Calappidae) Ciencia y Tecnología del Mar 10:33-59. 